A PRIMER IN ELASTICITY 


\section{A Primer in Elasticity}

by

PAOLO PODIO-GUIDUGLI

Department of Civil Engineering,

University of Rome "Tor Vergata",

Rome, Italy

Reprinted from Journal of Elasticity, Vol. 58, No. 1 (2000)

SPRINGER-SCIENCE+BUSINESS MEDIA, B.V. 
Library of Congress Cataloging-in-Publication Data

A C.I.P. Catalogue record for this book is available from the Library of Congress.

ISBN 978-90-481-5592-7 ISBN 978-94-017-0594-3 (eBook)

DOI 10.1007/978-94-017-0594-3

Printed on acid-free paper

\begin{abstract}
All Rights Reserved
(C2000 Springer Science+Business Media Dordrecht

Originally published by Kluwer Academic Publishers in 2000

No part of the material protected by this copyright notice may be reproduced or utilized in any form or by any means, electronic or mechanical, including photocopying, recording or by any information storage and retrieval system, without written permission from the copyright owner.
\end{abstract}


A Sara, Marta e Adriano 
Chapter I Strain

1. Deformation. Displacement

2. Rigid Deformations. Pure Strains

3. Strain Measures 11

4. Small Strain 14

5. Simple Deformations 17

6. Divergence Identities 19

Chapter II Stress

7. Forces. Balances 25

8. Stress. Dynamical Processes 30

9. Simple Equilibrium Solutions. Normal and Shear Forces 32

10. Alternative Forms of the Basic Balance Laws 35

11. Power. Stress Power 38

12. Exact and Linearized Equilibrium Theories 40

Chapter III Constitutive Assumptions

13. Linearly Elastic Materials 47

14. Material Symmetry 51

15. Fourth-Order Tensors 54

16. Problems of Classification and Representation 56

17. Internal Constraints $\quad 60$

18. Constraints and Material Symmetry 65

19. Interpretation of Material Moduli 70

Chapter IV Equilibrium

20. Classical, Strong, and Weak Formulations 75

21. Variational Formulation. The Principle of Minimum Potential Energy 80

22. Minimum Complementary Energy. Variational Principles 84

23. Compatible Field and Boundary Operators 90

24. Generalized Boundary Conditions 93

25. Elastic Equilibrium with the Cauchy Relations 96

26. Elastic Equilibrium in the Presence of Internal Constraints 98

$\begin{array}{ll}\text { References } & 103\end{array}$

$\begin{array}{ll}\text { Subject Index } & 105\end{array}$ 


\section{Preface}

Linear Elasticity is "the mother of all theories" in continuum physics. In the century now about to close its traditional devotees - the mathematician and the engineer have been joined by geophysicists, biomechanists, materials scientists, and other users. By a seeming paradox, the variety and disparity of current applications calls for a study of elasticity per se. This book, which is directed to both mathematicians and physical scientists, presents the foundational issues of linear elasticity in a compact, unabridged manner. Since its intended readership certainly includes that of the Journal of Elasticity, I have welcomed the opportunity I have been offered by Kluwer to reach that primary target by publishing this writing first in that journal, under form of a special presentation invited by the Editor-in-Chief, Professor R.L. Fosdick.

There are four chapters: the first two illustrate, respectively, the concepts of deformation and strain and of force and stress; the third is devoted to a study of constitutive relations; the last discusses the posing of equilibrium problems. The emphasis is in the description of elasticity as a model whose construction calls for a delicate interplay between physics and mathematics. The conceptual links with general continuum mechanics - in particular, with finite elasticity - are carefully indicated, especially when a notion, as is the case with strain and stress, is better understood when presented in full. As is to be expected when the goal is to discuss the foundations of a theory in a slim essay, no pretenses of comprehensiveness are made, but occasional concessions to personal taste are found.

This book could not be written without the knowledge gained from two masterful works by M.E. Gurtin, his Handbuch article on linear elasticity of 1972 and his Introduction to Continuum Mechanics of 1981. Its peculiarities are an updated presentation of the basic concepts and an unusual coverage: it would not be easy to find in one other book in elasticity a treatment of such issues as exact and linearized equilibria, the constitutive problems of classification and representation, internal constraints and material symmetries, elastic equilibrium with the Cauchy relations, and elastic equilibrium in the presence of internal constraints. As the title suggests, this is meant to be a short, condensed handbook to be used to teach. In fact, the book is an outgrowth of advanced undergraduate and graduate courses I have repeatedly given, in my own institution and elsewhere, for students in applied mathematics and engineering; for this reason, a score of figures and one hundred exercises of variable difficulty are included. 
I want to thank R.L. Fosdick, M.E. Gurtin and W.O. Williams for their detailed criticism of the manuscript. I also thank F. Davì, M. Lembo, P. Nardinocchi and M. Vianello for valuable remarks prompted by their reading of one or another of the many previous drafts, from 1988 to date. Since it has taken me so long to bring this writing to its present form, many other colleagues and students have episodically offered useful comments and caught mistakes: a list would risk to be incomplete, but I am heartily grateful to them all. Finally, I thank V. Nicotra for skillfully transforming my hand sketches into book-quality figures.

P. PODIO-GUIDUGLI

Roma, April 2000 